\title{
A POPULATION DISCRIMINANT IN M-DWARF SPECTRA
}

\author{
W. P. BIDELMAN \\ Case Western Reserve University, East Cleveland, U.S.A. \\ and \\ W. G. SMETHELLS \\ University of Wisconsin, Eau Claire, U.S.A.
}

\begin{abstract}
The $\mathrm{CaH}$ bands in the $\lambda 6385$ region of the spectra of $\mathrm{M}$ dwarfs have long been known; they become visible in the late $K$ 's and show a gradual increase in strength to later types. However, Greenstein and Eggen have noted that there is a substantial strengthening of all the hydride bands, including $\mathrm{CaH}$, in high-velocity dwarfs (notably G95-59), and one of us (W.P.B.) has also seen this effect in suitable Warner and Swasey Observatory objective-prism plates of the rather low-velocity subdwarfs Ross 730 and 731 . In a recent southern-hemisphere survey with the Curtiss Schmidt, Smethells has discovered nearly 200 late $\mathrm{K}$ and early $\mathbf{M}$ dwarfs, for all of which estimates of CaH-band strength were made. He found a considerable variation of the $\mathrm{CaH}$-band strength in stars of the same (TiO-band) spectral type, and a slight tendency for the $\mathrm{CaH} / \mathrm{TiO}$ band ratio to increase with increasing tangential velocity. A fuller account of this work will appear elsewhere.
\end{abstract}

\section{DISCUSSION}

McCarthy: My congratulations on the possible new metal abundance discriminant reported here. There are two other luminosity criteria available as discriminants, $\mathrm{Na} \mathrm{D}$ lines (as discovered by W. Luyten in 1923 ) and $\mathrm{MgH}$. Can you tell us if any correlations have been made with these?

Bidelman: $\mathrm{CaH}$ and $\mathrm{MgH}$ behave very similarly. As far as the $\mathrm{D}$ lines are concerned, we have used their strength as the primary discriminator between giants and dwarfs, but in view of their confusion with a strong TiO band I would think it difficult to use these for any other purpose. 